Research Article

\title{
A Retrospective Analysis of Multi-Drug Resistant Tuberculosis among Presumptive Pulmonary Drug-Resistant Tuberculosis Patients in Andaman \& Nicobar Islands
}

\author{
SP Burma', Keerthivasan Gurumurthy', Arjun Sivadas ${ }^{3}$
}

${ }^{1}$ Head of the Department, Department of Tuberculosis and chest Diseases, Andaman and Nicobar Islands Institute of Medical Sciences, Port Blair, Andaman and Nicobar Islands.

${ }^{2}$ Microbiologist- External Quality Assurance, State TB control Society, Port Blair, Andaman and Nicobar Islands. ${ }^{3}$ DOTS Plus Site Senior Medical Officer, State TB control Society, Port Blair, Andaman and Nicobar Islands.

DOI: https://doi.org/10.24321/2454.8642.201904

$\begin{array}{lllllll} & \mathbf{N} & \mathbf{F} & \mathbf{O}\end{array}$

Corresponding Author:

Keerthivasan Gurumurthy, MicrobiologistExternal Quality Assurance, State TB control Society, Port Blair, Andaman and Nicobar Islands. E-mail Id:

keerthivasan.g2@gmail.com

Orcid Id:

https://orcid.org/0000-0003-4509-3741

How to cite this article:

Burma SP, Gurumurthy K, Sivadas A. A Retrospective Analysis of Multi-Drug Resistant Tuberculosis among Presumptive Pulmonary Drug-Resistant Tuberculosis Patients in Andaman \& Nicobar Islands. Rec Adv Path Lab Med 2019; 5(2): 1-4.

Date of Submission: 2019-07-10

Date of Acceptance: 2019-08-02

\section{$\begin{array}{llllllll}\mathbf{A} & \mathbf{B} & \mathbf{S} & \mathbf{T} & \mathbf{R} & \mathbf{A} & \mathbf{C} & \mathbf{T}\end{array}$}

Introduction: Tuberculosis is a contagious bacterial infection affecting more than 10 million people worldwide. It is a major public health concern in India especially with the increasing numbers of drug-resistant cases of tuberculosis. Lack of early diagnosis, inadequate and inappropriate treatment are the major contributors to the development of drug resistance. PMDT strategy strives for early diagnosis and appropriate treatment of drug-resistant TB. This study was undertaken to estimate the rate of occurrence of MDR-TB among presumptive DR-TB patients in Andaman \& Nicobar Islands.

Materials and Methods: A retrospective data based study for the calendar year 2018 was done by the state TB centre of the Union Territory of Andaman and Nicobar Islands. All patients with presumptive pulmonary DR-TB (as defined by the PMDT guidelines 2017) were included in the study. The drug resistance was confirmed by phenotypic and genotypic methods.

Result: The total number of presumptive DR-TB cases during the calendar year 2018 was 235 . Forty eight of the cases were confirmed to be MDR-TB cases. Additional drug resistance to Second Line Injectable aminoglycosides (SLI) and Fluoroquinolones (FQ) was found in 2 and 6 of the cases respectively. Two cases of extreme drug resistant tuberculosis were also found (MDR+SLI+FQ resistance).

Conclusion: The best strategy for the control of drug resistant tuberculosis is prevention, which can only be achieved by timely diagnosis and early initiation of appropriate treatment of drug resistant TB cases as per drug susceptibility results, thereby preventing their further transmission.

Keywords: MDR-TB, Andaman and Nicobar Islands, DR-TB, CBNAAT 


\section{Introduction}

Tuberculosis (TB) is one of the major causes of mortality and morbidity globally. India being the second most populous country, contributes almost a quarter of the global tuberculosis burden. The estimated incidence of tuberculosis in India in 2016 was 27, 90, 000. ${ }^{1}$ Multi-drug resistant tuberculosis (MDR-TB) is a serious public health concern worldwide including India. MDR-TB is defined as resistance to both Isoniazid $(\mathrm{H})$ and Rifampicin (R) with or without resistance to other anti-TB drugs. ${ }^{2}$ The estimated incidence of MDR-TB in India in 2016 was 1, 47, 000 which accounts for one-fourth of the total cases world-wide. ${ }^{1}$

The Revised National Tuberculosis Control Program (RNTCP), an initiative of Government of India formulates policies and strategies to provide quality preventive, diagnostic and therapeutic services for tuberculosis in India since 1998. Emergence of MDR-TB over the past few decades is mainly attributed to inadequate or inappropriate anti tubercular regimen. The treatment success rate among notified drug-resistant TB (DR-TB) cases is $46 \%$ as compared to $75 \%$ success rate among drug sensitive TB (DS-TB) cases. ${ }^{1,3}$ This necessitates aggressive approach to combat the rising numbers of DR-TB cases in India. Programmatic Management of Drug-resistant TB (PMDT) began in 2007 and national coverage was achieved by $2013 .^{2}$ Its main objective is early diagnosis and appropriate treatment of drug-resistant TB (DR-TB), thereby minimizing the spread of DR-TB. In Andaman \& Nicobar Islands PMDT services began in 2012.

The drug susceptibility testing (DST) for anti-tubercular drugs is done by phenotypic methods (solid and liquid culture) and genotypic methods (Cartridge Based Nucleic Acid Amplification Test, Line Probe Assay, Polymerase Chain Reaction etc.)

The Andaman and Nicobar group of Islands is an archipelago of more than 300 islands in the Bay of Bengal with a population of about 3.81 lakhs. (2011 Census report). In this study we sought to determine the rate of occurrence of MDR-TB among presumptive DR-TB patients in the islands.

\section{Materials and Methods}

The study was conducted by the State TB Centre of Andaman and Nicobar Islands. Retrospective record based study was done for the period, January 2018 to December 2018. All patients with presumptive pulmonary DR-TB (as defined by the PMDT guidelines 2017) were included in the study.

Presumptive pulmonary DR-TB cases were referred to the nodal DR-TB centre/ district DR-TB centres from the peripheral health institutions of all the three districts of Andaman and Nicobar Islands, as per PMDT guidelines. Gene-Xpert test was done in CBNAAT sites across the islands. Culture and first/ second- line LPA were done in the Intermediate Reference Laboratory, Chennai.

\section{Result}

The total number of presumptive DR-TB cases during the calendar year 2018 was 235 . Among the total cases, 43 were previously treated tuberculosis patients, 5 were TB patients who were contacts of DR-TB cases, 2 were new TB patients with HIV co-infection, 1 a paediatric TB non responder, 6 TB patients with treatment failure and rest were other notified new TB cases (Figure 1).

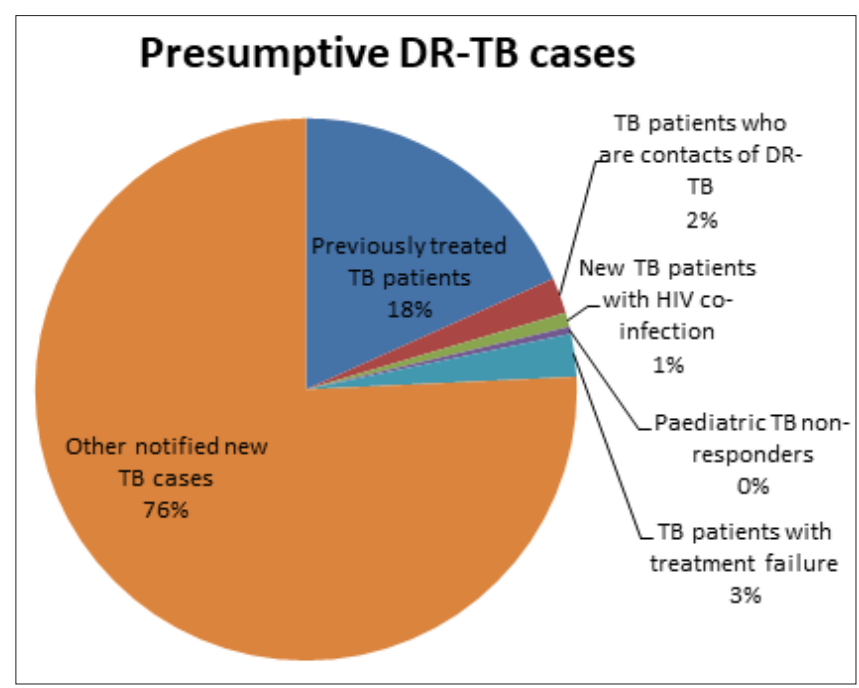

Figure I.Presumptive DR-TB cases

Forty eight were confirmed to be MDR-TB cases. Additional drug resistance to Second Line Injectable aminoglycosides (SLI) and Fluoroquinolones (FQ) was found in 2 and 6 cases of the cases respectively. Two cases of extreme drug resistant tuberculosis were also found (MDR+SLI+FQ resistance) (Figure 2).

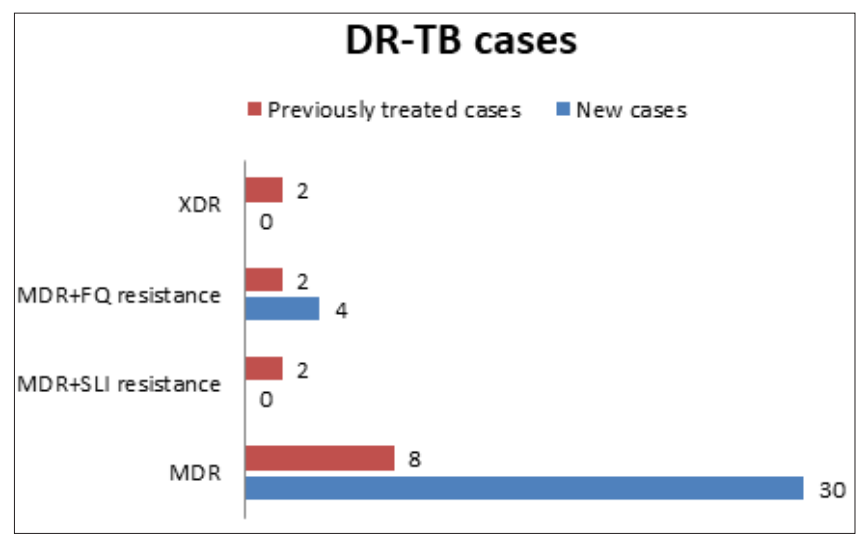

Figure 2.Drug resistant tuberculosis cases

There are 3 districts in Andaman and Nicobar Islands South Andaman, North and Middle Andaman and Nicobar. The district-wise distribution of DR-TB cases is depicted in figure 3. 


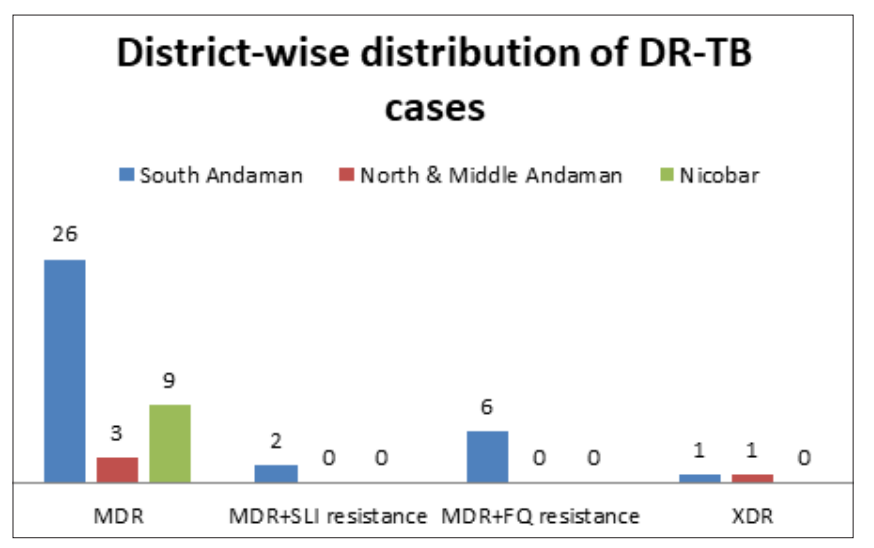

Figure 3.District-wise distribution of DR-TB cases

Demographic profile of the DR-TB cases is depicted in figure 4.

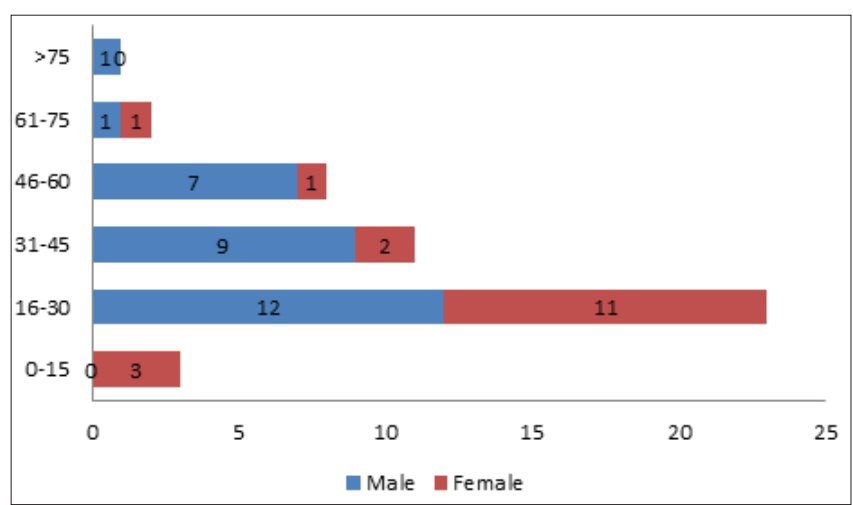

Figure 4.Demographic profile of DR-TB cases

\section{Discussion}

Globally more than 10 million people are affected by tuberculosis, with India harbouring one fourth of the cases. ${ }^{1}$ Tuberculosis is a curable disease with appropriate treatment regimen and preventive measures. Thus WHO has initiated the END TB strategy to significantly reduce TB mortality and morbidity by 2035 . But mismanagement of anti tubercular treatment has led to the emergence of MDR-TB strains. The treatment of drug sensitive tuberculosis has a higher success rate and is more cost effective. ${ }^{4}$ The rising incidence of drug resistant tuberculosis cases has put considerable strain on the public health and economy of developing countries like India.

The PMDT initiative through RNTCP was established to tackle the menace of drug resistant tuberculosis.

This study was done to ascertain the rate of occurrence of MDR-TB in the Andaman and Nicobar Islands. A total of 235 cases of presumptive DR-TB were included in the study. Out of these, 48 were confirmed to be MDR-TB cases. A large number of these cases were young adults in the age group of $16-30$ years (68.8\%). An epidemiological study of MDR-TB from Ahmedabad also showed similar findings. ${ }^{5}$ Several other studies also have noted increased prevalence of drug resistance among young adults..$^{6-8}$ Drug resistance was noted to be higher among male patients (62.5\%). This is in accordance with other recent studies from India. 5,9,10

Out of the 235 cases of presumptive DR-TB, 48 were confirmed to be positive for multi-drug resistance (20.4\%) which is much higher than the national average of around $6 \% .^{2}$ This is also in contrast to many other studies from mainland India suggesting a prevalence closer to the national average. ${ }^{4,11}$ But such a high rate is not uncommon as a study from New Delhi has also noted the prevalence of MDR-TB to be $20.4 \% .{ }^{8}$ Out of these 48 MDR-TB cases, 34 were newly detected tuberculosis cases implying a higher incidence of primary MDR-TB. Primary MDR-TB refers to patients infected with Mycobacterium tuberculosis that is resistant to anti tubercular drugs before initiation of treatment. Among the MDR-TB cases, additional resistance to $\mathrm{SLI}$ and FQ were noted in $4.2 \%$ and $12.5 \%$ of the cases respectively. This is comparable to the national statistics. The rate of occurrence of XDR-TB was ascertained to be $4.2 \%$ which is higher than the national average. This higher prevalence of resistance could be due to the geographical and demographical challenges unique to these islands.

The above mentioned figures paint an alarming picture of drug resistance in tuberculosis. But, in reality it may be underestimating the true burden, as this study has not included smear-negative pulmonary TB and extrapulmonary TB. In addition, there is lack of involvement in TB notification and non-adherence to recommended treatment regimens by private sector. This is also true of the conclusions derived from other similar studies. ${ }^{12-14}$

The Union Territory of Andaman and Nicobar is a group of islands in the Bay of Bengal more than 1000 kilometres away from mainland India. It is divided into three administrative districts-North and Middle Andaman, South Andaman and Nicobar, each having a district tuberculosis centre. There are 15 designated microscopy centres and 4 CBNAAT sites dedicated towards TB diagnosis. The challenging topographical nature of these islands and inadequate transport facilities makes it difficult for the patients to avail timely diagnostic and therapeutic services. Increasing influx of migrant labourers catering to various developmental projects makes the disease surveillance and follow up an uphill task and crowded living conditions compound to the problem. Due to the unavailability of laboratory infrastructures rendering services like culture and drug susceptibility testing of first line and second line anti tubercular drugs, samples are being sent to IRL Chennai (1400 Kms apart). This results in a considerable delay in the transport of specimens from peripheral health centres to the laboratory resulting in an increased rate of sample contamination, further delaying definitive diagnosis. The newer treatment regimens of MDR-TB comprising drugs 
like Bedaquiline and Delamanid also warrant access to a laboratory with expanded second-line DST. Thus, initiating treatment regimens with these newer drugs also poses a challenge in these islands, underlying the need for a wellequipped laboratory in the state head quarters. ${ }^{15}$

\section{Conclusion}

World Health Organization (WHO) has initiated "END TB" strategy to drastically reduce the global TB burden by 2035. India bearing the majority burden has taken up this challenge with even more vigour to achieve this goal by 2025. This can only be achieved by curtailing the rising numbers of DR-TB cases which have a significantly lower treatment success rate. Hence, PMDT guidelines must be strictly adhered to, in order to achieve early diagnosis and appropriate treatment thereby preventing further transmission. A multi-faceted approach towards improving the accessibility of diagnostic laboratories, dedicated drugresistant TB treatment centres, availability of newer anti DR-TB drugs, increasing public awareness and patient compliance is the need of the hour.

\section{Acknowledgements: None \\ Conflict of Interest: None \\ References}

1. Annual Reports: Central TB Division, TB India Report 2018. [cited 2019 Jul 5]. Available from: https:// tbcindia.gov.in/index1.php.

2. Guideline for PMDT in India 2017 : Central TB Division. [cited $2019 \mathrm{Jul}$ 5]. Available from: https://tbcindia.gov. in/index1.php.

3. Dhole T, Maurya A, Singh A et al. Changing Patterns and Trends of Multidrug-resistant Tuberculosis at Referral Centre in Northern India: A 4-year Experience. Indian J Med Microbiol 2013; 31(1): 40-46.

4. Sk B, Gc A, Preeti P et al. Prevalence of MDR-TB among New and Previously Treated Patients of Pulmonary Tuberculosis in DRTB Centre of Faridkot - A Retrospective Study. International Journal of Contemporary Medical Research 2017;4(6): 1283-1286.

5. Bhatt G, Vyas S, Trivedil K. An Epidemiological Study of Multi drug resistant Tuberculosis Cases Registered under Revised National Tuberculosis Control Programme of Ahmedabad City. Indian J Tuberc 2012; 59(1): 18-27.

6. Shah A, Shah R, Dave P. Factors contributing to Development of Multidrug Resistant Tuberculosis. Natl J Physiol Pharm Pharmacol 2018; 8(10): 1463-1469.

7. Gaude GS, Hattiholli J, Kumar P. Risk Factors and Drugresistance Patterns Among Pulmonary Tuberculosis Patients in Northern Karnataka region, India. Niger Med J J Niger Med Assoc 2014; 55: 327-332.

8. Sharma SK, Kumar S, Saha PK et al. Prevalence of Multidrug-resistant Tuberculosis Among Category II
Pulmonary Tuberculosis Patients. Indian J Med Res 2011; 133: 312-315.

9. Shri Ram Ganesh RT, Manimaran Namasivayam K, Uma $\mathrm{N}$ et al. A Retrospective Study Risk Factor Analysis in MDR TB Cases Among Rural Population of Thanjavur Medical College and Hospital, Thanjavur, Tamilnadu. J Evid Based Med Healthc 2018; 5: 1211-1215.

10. Venkatesh U, Srivastava D, Srivastava A et al. Epidemiological Profile of Multidrug-resistant Tuberculosis Patients in Gorakhpur Division, Uttar Pradesh, India. J Fam Med Prim Care 2018; 7(3): 589595.

11. Kumar A, Singh A, Upadhyay V et al. Epidemiology of Multi-drug-resistant tuberculosis in Northern India. Biomed Biotechnol Res J BBRJ 2018; 2(2): 112-121.

12. Arinaminpathy $N$, Batra $D$, Khaparde $S$ et al. The Number of Privately Treated Tuberculosis Cases in India: An Estimation from Drug Sales Data. Lancet Infect Dis 2016; 16(11): 1255-1260.

13. Dalal A, Pawaskar A, Das M et al. Resistance Patterns Among Multidrug-resistant Tuberculosis Patients in Greater Metropolitan Mumbai: Trends Over Time. PloS One 2015; 10(1): e0116798.

14. Satyanarayana S, Subbaraman R, Shete P et al. Quality of Tuberculosis Care in India: a Systematic Review. Int J Tuberc Lung Dis Off J Int Union Tuberc Lung Dis 2015; 19(7): 751-763.

15. Pothula M, Reddy S, Vasundhara P et al. Study of Prevalence of Multi-Drug Resistant Tuberculosis in a Tertiary Care Hospital. BMC Infect Dis 2012; 12(Suppl 1): P29. 